\title{
artigo
}

Fernandes, V.M.B.; Santos, E.K.A.; Backes, M.R.S.; Rea, M.F.; Araújo, R.G.; Iglesias, J.S.P.;

A prática do aleitamento materno entre as trabalhadoras formais: revisão integrativa de literatura

DOI: https://doi.org/10.36489/saudecoletiva.2020v10i58p4141-4052

\section{A prática do aleitamento materno entre as trabalhadoras formais: revisão integrativa} de literatura

The practice of breastfeeding among female formal workers: integrative literature review La práctica de la lactancia materna entre las formales trabajadoras: revisión integradora de la literatura

\begin{abstract}
RESUMO
Objetivo: identificar a contribuição das pesquisas desenvolvidas, em âmbito nacional e internacional, acerca da prática do aleitamento materno entre trabalhadoras formais. Método: revisão Integrativa de Literatura, com 18 artigos científicos, coletados em seis fontes de dados: CINAHL, LILACS, BDENF, SciELO, Scopus e Medline/PubMed, publicados no período de 2010 a 2014, analisados através da Análise de Conteúdo do tipo categorial temática de Laurence Bardin, com auxílio do software Atlas.ti. Resultados: evidenciou-se duas categorias: tipos de apoio durante a manutenção da amamentação e ambiente de trabalho amigo da trabalhadora que amamenta. Conclusão: a produção científica nesta temática é escassa, porém, os estudos demonstram que apesar das dificuldades enfrentadas após o retorno ao trabalho da licença-maternidade, os resultados revelam que existe a possibilidade de conciliar trabalho com amamentação desde que haja uma rede de apoio bem estabelecida e um local de trabalho que apoia a amamentação além daquilo que é exigido pelas legislações.

DESCRITORES: Aleitamento materno; Mulheres trabalhadoras; Legislação Trabalhista; Políticas Públicas de Saúde; Organizações.
\end{abstract}

\section{ABSTRACT}

Objective: to identify the contribution of research carried out, nationally and internationally, on the practice of breastfeeding among formal workers. Method: Integrative Literature review, with 18 scientific articles, collected from six data sources: CINAHL, LILACS, BDENF, SciELO, Scopus and Medline / PubMed, published between 2010 and 2014, analyzed through the Content Analysis of the categorical type Laurence Bardin theme, with the help of Atlas.ti software. Results: there were two categories: types of support during breastfeeding maintenance and a working environment that is friendly to the breastfeeding worker. Conclusion: scientific production on this topic is scarce, however, studies show that despite the difficulties faced after returning to work on maternity leave, the results reveal that there is a possibility of reconciling work with breastfeeding as long as there is a well-supported support network. established and a workplace that supports breastfeeding beyond what is required by legislation.

DESCRIPTORS: Breast Feeding; Women, working; Legislation, Labor; Public Health Policy; Organizations.

\section{RESUMEN}

Objetivo: identificar el aporte de las investigaciones realizadas, a nivel nacional e internacional, sobre la práctica de la lactancia materna en trabajadoras formales. Método: Revisión de Literatura Integrativa, con 18 artículos científicos, recolectados de seis fuentes de datos: CINAHL, LILACS, BDENF, SciELO, Scopus y Medline / PubMed, publicados en el período de 2010 a 2014, analizados mediante Análisis de Contenido categórico. Tema Laurence Bardin, con la ayuda del software Atlas.ti. Resultados: hubo dos categorías: tipos de apoyo durante el mantenimiento de la lactancia y un ambiente de trabajo amigable para la trabajadora que amamanta. Conclusión: la producción científica sobre este tema es escasa, sin embargo, los estudios muestran que a pesar de las dificultades que se enfrentan luego de la reincorporación al trabajo en baja por maternidad, los resultados revelan que existe la posibilidad de conciliar el trabajo con la lactancia materna siempre que se cuente con una red de apoyo bien sustentada. establecido y un lugar de trabajo que apoye la lactancia materna más allá de lo que exige la legislación.

DESCRIPTORES: Lactancia Materna; Mujeres trabajadoras; Legislación Laboral; Políticas Públicas de Salud; Organizaciones.

RECEBIDO EM: 21/08/2020 APROVADO EM: 14/09/2020 


\section{Vanessa Martinhago Borges Fernandes}

Enfermeira. Doutoranda em Enfermagem pelo Programa de Pós-Graduação de Enfermagem da Universidade Federal de Santa Catarina - UFSC. Docente da Universidade do Sul de Santa Catarina - UNISUL. Palhoça, SC, Brasil.

ORCID: 0000-0002-0552-7229

\section{Evangelia Kotzias Atherino dos Santos}

Enfermeira. Doutora em Enfermagem. Docente do Programa de Pós-Graduação de Enfermagem da Universidade Federal de Santa Catarina - UFSC. Florianópolis, SC, Brasil.

ORCID: 0000-0002-5970-020X

\section{Marli Terezinha Stein Backes}

Enfermeira Obstétrica. Doutora em Enfermagem. Docente do Departamento de Enfermagem no Curso de Graduação em Enfermagem, da Pós-Graduação em Enfermagem e do Programa de Pós-Graduação de Gestão do Cuidado em Enfermagem da Universidade Federal de Santa Catarina - UFSC. Florianópolis, SC, Brasil.

ORCID: 0000-0003-3258-359X

\section{Marina Ferreira Rea}

Médica. Doutora em Medicina Preventiva pela USP. Pesquisadora-sênior (VI) do Instituto de Saúde -SES-SP - aposentada; membro da Coordenação Internacional da Rede IBFAN. São Paulo, SP, Brasil.

ORCID: 0000-0003-1540-7973

\section{Renara Guedes Araújo}

Nutricionista. Mestre em Saúde da Mulher e da Criança pelo Instituto Fernandes Figueira - Fundação Oswaldo Cruz. Consultora Técnica do Ministério da Saúde na Coordenação de Saúde da Criança e Aleitamento Materno. Brasília, DF, Brasil.

ORCID: 0000-0001-7057-9458

\section{Jessicamila dos Santos Pais Iglesias}

Enfermeira. Especialista em Saúde da Família pelo Programa de Residência Multiprofissional PMF/UDESC/PREMULTISF. Enfermeira do Núcleo Médico Clínico do Hospital SOS Cárdio. Florianópolis, SC, Brasil.

ORCID: 0000-0003-0833-207X

\section{INTRODUÇÃO}

A revolução industrial foi um momento histórico conhecido como responsável pelo desmame precoce no mundo do século XIX até o século XXI, devido a entrada em massa das mulheres no mercado de trabalho ${ }^{(1)}$. Na idade contemporânea as estruturas familiares se reconfiguraram e houve avanços na inserção da mulher no mercado de trabalho. Portanto, torna-se inevitável a reorganização dos processos de trabalho para a conciliação com as funções maternas, o que vai ao encontro dos benefícios mútuos e da prevenção de morbidades ${ }^{(2)}$.

Apesar dos avanços, nenhum país no mundo atende as recomendações de investimento financeiro direcionados às ações de amamentação e somente 23 países atingiram a meta global de 2030 de alcançar a taxa de $60 \%$ para o aleitamento materno exclusivo ${ }^{(3)}$.
A dificuldade

em amamentar

e trabalhar é

apresentado como

motivo para que

mulheres deixem

seus empregos...
A dificuldade em amamentar e trabalhar é apresentado como motivo para que mulheres deixem seus empregos, em contrapartida, estudos demonstram que é mais comum que elas permaneçam no trabalho, utilizem leites artificiais ou desmamem precocemente. As trabalhadoras não recebem apoio adequado para conciliar trabalho com amamentação, as que trabalham em regime informal nem ao menos possuem direitos trabalhistas ${ }^{(4)}$.

As evidências corroboram para a primordialidade em abranger a prática profissional para além dos aspectos biológicos da amamentação, compreendendo toda a complexidade que envolve esta prática e considerando as dimensões multifacetadas que envolvem a mulher como mãe, nutriz, esposa, trabalhadora e cidadã, contribuindo para que a amamentação seja satisfatória para o binômio mãe-filho ${ }^{(5)}$.

A fim de gerar um panorama sobre a 


\section{artigo}

Fernandes, V.M.B.; Santos, E.K.A.; Backes, M.R.S.; Rea, M.F.; Araújo, R.G.; Iglesias, J.S.P.;

A prática do aleitamento materno entre as trabalhadoras formais: revisão integrativa de literatura

prática de amamentação de trabalhadoras formais (mulheres que possuem direitos trabalhistas) e conhecer como trabalhadoras de outros lugares do mundo fazem para conciliar trabalho e amamentação, desenvolvemos a presente revisão integrativa de literatura (RIL), tendo como pergunta norteadora: Qual o conhecimento científico produzido na literatura nacional e internacional sobre a trabalhadora formal que amamenta? E como objetivo: Identificar a contribuição das pesquisas desenvolvidas, em âmbito nacional e internacional, acerca da prática do aleitamento materno entre trabalhadoras formais.

\section{MÉTODO}

Trata-se de uma RIL, método de investigação que realiza análise crítica, compila evidências científicas sobre uma temática e identificando lacunas, seguindo seis etapas segundo o fluxograma a seguir $^{(6)}$, com protocolo elaborado pela autora principal e conferência do checklist PRISMA.

$\mathrm{Na} 1^{\text {a }}$ etapa foi realizada a identificação do tema, elaborados a questão de pesquisa e o objetivo supracitados. Para a coleta dos artigos foram definidos os descritores, conforme os Descritores em Ciências da Saúde (DeCS): aleitamento materno/breast feeding/lactancia materna e trabalho feminino/working, women/trabajo de mujeres. $\mathrm{Na} 2^{a}$ etapa foram estabelecidos os critérios de inclusão: publicações com limite temporal entre os anos de 2010 a 2014. Dentre os critérios de exclusão estavam todos os tipos de estudos que não se caracterizassem como artigo científico. Foram selecio-

\section{Figura 1 - Descrição dos estudos incluídos na RIL}

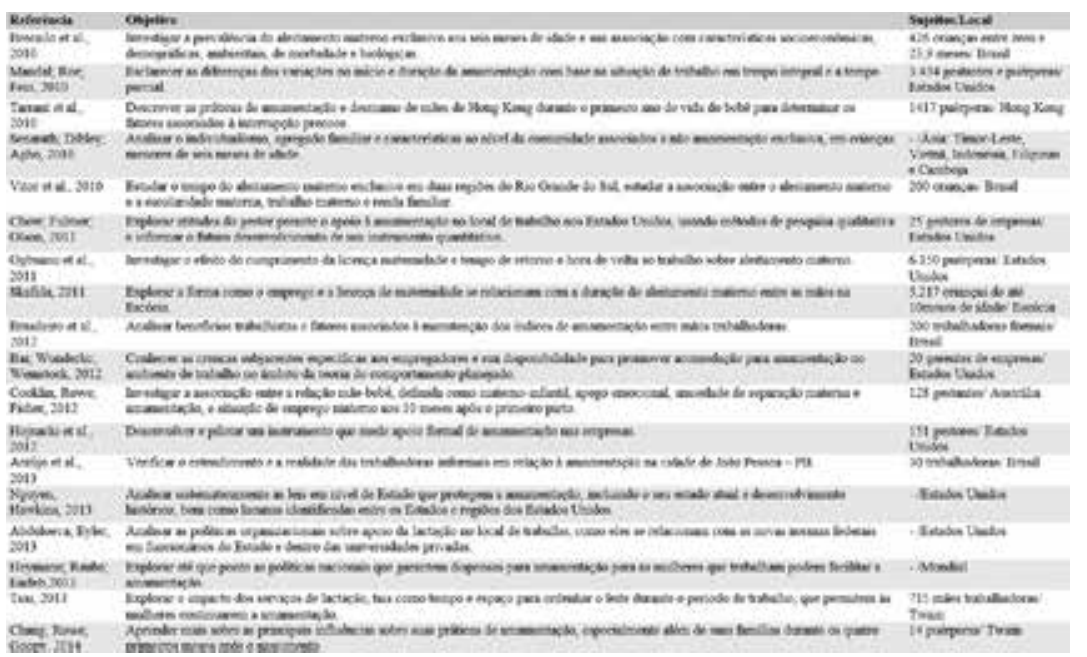

Fonte: autora, 2015

\section{Figura 2: Tipos de apoio durantes a manutenção da amamentação}

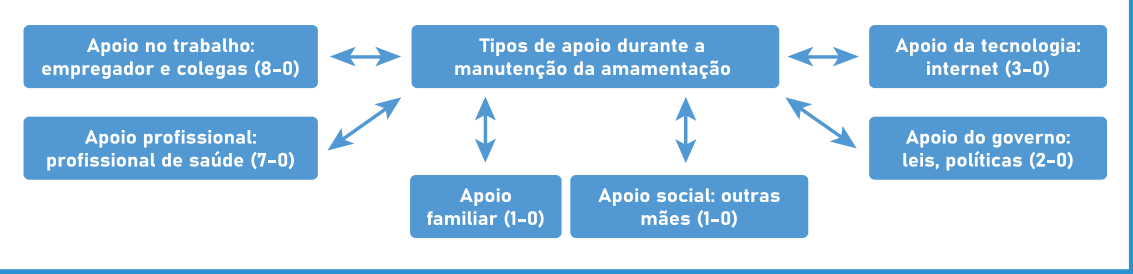

Fonte: autora, 2015 nadas seis fontes de dados: CINAHL, LILACS, BDENF, SciELO, Scopus e Medline/PubMed. Foram coletados um total de 125 artigos. Na $3^{\text {a }}$ etapa foram definidas as informações a serem extraídas dos estudos selecionados, a organização dessas informações e a formação de um banco de dados, utilizando o Programa Endnote para armazenamento e organização das referências bibliográficas e o Programa Microsoft Office Word 2007 para organizar as informações retiradas dos materiais. Para a $4^{a}$ etapa foi realizada a avaliação dos artigos, realizando a inclusão e exclusão através da leitura geral e seleção de 18 estudos. $\mathrm{Na} 5^{a}$ etapa foi realizada a interpretação dos resultados através do Programa Atlas.ti, na versão 7.0, que auxilia na análise de dados de pesquisas qualitativas. A $6^{a}$ etapa consistiu na apresentação dos resultados em forma de manuscrito.

\section{RESULTADOS}

A síntese dos resultados evidenciou duas categorias e a descrição dos estudos está apresentada na figura a seguir:

\section{Categoria 1: Tipos de apoio durante a manutenção da amamentação}

O apoio familiar é fonte de encorajamento na construção do vínculo entre a mãe e seu filho ${ }^{(7)}$. Além disso, a possibilidade de desmame precoce é maior naquelas que não participam de programas de incentivo à amamentação ou não obtém apoio profissional ${ }^{(7,8)}$.

A assistência dos profissionais de saúde foi relacionada com a melhoria da amamentação na Indonésia ${ }^{(9)}$. Em Taiwan, permanecer em centro de cuidados no primeiro mês pós-parto influenciou positivamente na prática, devido apoio e aconselhamento de profissionais, especialmente enfermeiros ${ }^{(10)}$. E mães de Hong Kong, retornam ao trabalho e recebem educação profissional adicional ${ }^{(11)}$.

Porém, nem todas as mulheres retornam ao trabalho devido à falta de apoio $^{(10,12-13)}$. Alguns empregadores têm atitudes positivas em relação ao provi- 
mento de um ambiente favorável à mãe no trabalho. No entanto, é clara a percepção daqueles que se opõem e que defendem crenças de desvantagens para a empresa ${ }^{(14)}$.

O apoio da tecnologia, através da internet, encaixa-se no estilo de vida contemporâneo, incluindo sites não-interativos e interativos, sendo um recurso flexível, de anonimato, com muitas informações e de apoio social através de grupos de mães, que as motivam a amamentar e compartilhar suas experiências ${ }^{(10)}$.

O governo, através do apoio nas legislações e políticas, deve fornecer suporte para que as mães possam continuar amamentando $^{(11)}$, isso têm reduzido as barreiras em alguns locais de trabalho, mesmo nos Estados Unidos, onde a maioria das empresas ainda não tem programas formais de apoio à amamentação ${ }^{(16)}$. A segurança no emprego pode desempenhar papel importante em retardar o retorno ao trabalho após o parto ${ }^{(17)}$.

\section{Categoria 2: Ambiente de trabalho amigo da trabalhadora que ama- menta}

As trabalhadoras podem sentir-se desconfortáveis ao solicitar locais para amamentar ${ }^{(15)}$. Para que esse benefício tenha êxito, ela precisa receber treinamento para ordenhar, armazenar e oferecer seu leite adequadamente ${ }^{8}$. Um espaço privado para mulheres lactantes é um pequeno investimento, a sensibilização dos gestores pode acalmar sua incerteza de custo-efetivida$\mathrm{de}^{(14)}$, pois eles se preocupam com sua funcionalidade, sugerindo que a sala seja de multipropósito ${ }^{(14,18)}$. Todavia, o ambiente que não é exclusivo leva à cessação da amamentação ${ }^{(13)}$.

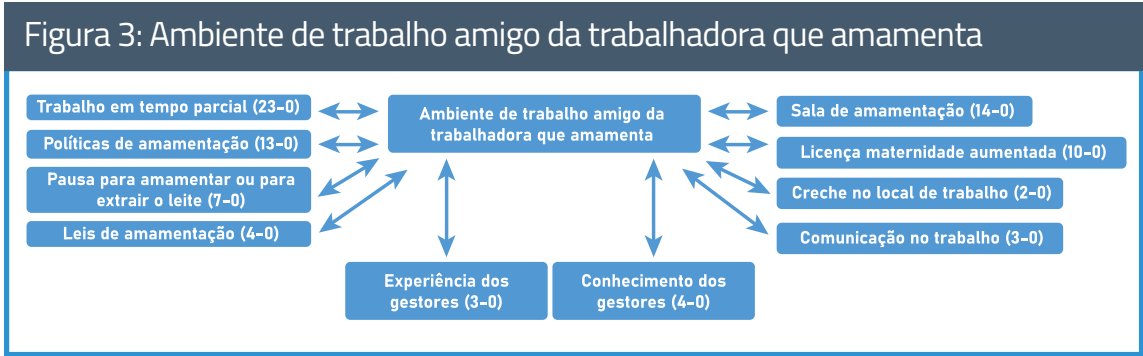

Fonte: autora, 2015

\section{O apoio da}

tecnologia, através

da internet, encaixa-

se no estilo de vida

contemporâneo,

incluindo sites

não-interativos e

interativos, sendo

um recurso flexível,

de anonimato, com

muitas informações

e de apoio social

através de grupos de

mães, que as motivam

a amamentar $\mathrm{e}$

compartilhar suas

experiências.
As mulheres também manifestam a insuficiência de pausas para realizar a extração do leite materno ${ }^{(19)}$. As chances do desmame antes do sexto mês são maiores do que para as mães que possuem o direito da pausa para amamentar ${ }^{(8,20)}$. Entretanto, a mulher pode precisar de um intervalo maior, políticas sugerem que ela possa utilizar o tempo de férias, além de realizar acordos como licença sem vencimento ou combinar horários flexíveis ${ }^{(21)}$.

Outra forma de acordo é o retorno ao trabalho em tempo parcial ${ }^{(18)}$. Estudos demonstram que o declínio na intensidade da amamentação é menor em relação ao retorno da mulher ao trabalho em tempo integral ${ }^{(13,16,18)}$.

A trabalhadora que possui uma licença-maternidade remunerada aumentada possui maiores chances de manter a amamentação ${ }^{(8,12,18,23)}$, devendo ser de cumprimento adequado ${ }^{(9,19)}$. Em contrapartida, um dos estudos revelou que as mulheres acreditam que o trabalho não traz influência no desmame, pois as mesmas não desistiram de amamentar após o regresso ${ }^{(11)}$.

Outro fator que aumenta a ansiedade da mulher, e da família como um todo, é o acesso limitado às creches ${ }^{(19)}$. A amamentação requer estreita proximidade ao longo do dia de trabalho, por isso é necessário que se instituam creches no trabalho ou próximas a ele ${ }^{(12)}$.

Políticas efetivas podem influenciar e acelerar o processo de manutenção da amamentação ${ }^{(21)}$. Campanhas para aumentar a conscientização do público sobre a importância e os direitos legais das mulheres relacionados com amamentação podem começar a mudar as normas sociais em torno da amamentação ${ }^{(24)}$. Além do conhecimento dos gestores em relação à amamentação, a experiência também pode influenciar em suas atitudes ${ }^{(15)}$. As empresas de modo geral não estão muito abertas para receberem pedidos de apoio à amamentação ${ }^{(18)}$. A minoria delas tem política escrita de amamentação. A criação dessa política pode ajudar a definir um ambiente e expectativa de apoio ${ }^{(18)}$, aumentando significativamente a chance da trabalhadora continuar amamentando ${ }^{(12-13)}$. 


\section{DISCUSSÃO}

$\mathrm{O}$ apoio familiar traz significativa influência sobre a manutenção da amamentação, assim como outros tipos de apoio não-familiares. $\mathrm{O}$ apoio à trabalhadora que amamenta é fundamental para que o processo se desenvolva de forma harmoniosa $^{(25)}$. As trabalhadoras destacam a necessidade de apoio por parte dos profissionais de saúde, o acolhimento e escuta qualificada podem produzir segurança e confiança na manutenção da amamentação. Também as redes de apoio informal promovem oportunidade para troca de experiência e conhecimento, grupos de puérperas e consultoria em amamentação podem ser estratégias inovadoras e são pouco utilizadas ${ }^{(26)}$.

A amamentação não se configura numa responsabilidade somente da mulher, é essencial buscar caminhos conjuntamente, objetivando a sensibilização dos gestores e discussão sobre as condições de trabalho, legislações e políticas ${ }^{(27)}$.

Dentro dos locais de trabalho podem encontrar-se ou não resistências em relação ao apoio à trabalhadora que amamenta, podem ser condutas prejudiciais: o desconhecimento e descumprimento de algumas legislações já conquistadas pelo Brasil, desconhecimento da situação de suas trabalhadoras, inexistência de política escrita de $\mathrm{AM}$ na empresa, o não suprimento de informações adequadas durante todo processo de maternidade, a opinião negativa de alguns gestores sobre o fornecimento de espaço adequado para ordenha do leite materno, tal como a sala de apoio à amamentação, não fornecimento de creche para filhos de trabalhadoras que amamentam, provimento de licença-maternidade de no máximo 120 dias consequência da não adesão ao Programa Empresa Cidadã, inflexibilidade de horários, entre outras ${ }^{(28)}$.

Além da rede de apoio completa, um conjunto de fatores essenciais foi detectado para que ao retornar ao trabalho a mãe possa encontrar um ambiente amigo da trabalhadora que amamenta. No Brasil, a partir de 2010, ações mais específi-
Dentro dos locais de trabalho podem

encontrar-se ou

não resistências em

relação ao apoio

à trabalhadora

que amamenta, podem ser condutas prejudiciais: 0 desconhecimento e descumprimento de algumas legislaçôes já conquistadas pelo Brasil, desconhecimento da situação de suas trabalhadoras, inexistência de política escrita de AM na empresa... cas relativas ao apoio à trabalhadora que amamenta foram implementadas no país, como incentivo à implantação de salas de apoio à amamentação em empresas, ao fornecimento de creche em todo território nacional e ao aumento da licença-maternidade para 180 dias $^{(29)}$.

No Brasil, as salas de apoio à amamentação não são obrigatórias por lei. A duração da licença-maternidade no Brasil é de no mínimo 120 dias $^{(30)}$, as empresas privadas, através do Programa Empresa Cidadã, podem aumentá-la para 180 dias ${ }^{(31)}$, e todas servidoras públicas federais e estaduais possuem o direito a 180 dias $^{(32)}$. A pausa para amamentar é garantida por lei somente para o servidor em regime celetista, fornecendo duas pausas de meia hora cada uma até que a criança complete seis meses de idade, configurando-se num tempo inadequado ${ }^{(33)}$. Quanto às creches, o regime celetista obriga toda empresa com pelo menos 30 mulheres com mais de 16 anos de idade a terem creche ou convênios durante o período da amamentação ${ }^{(33)}$. Sem comprometimento e investimento dinâmico o apoio à amamentação continuará inapropriado, imputando consequências negativas às gerações futuras ${ }^{(4)}$.

\section{CONCLUSÃO}

O presente estudo identificou que os estudos nesta temática são escassos, ainda que os resultados demonstrem a viabilidade na combinação entre as atividades de trabalhar e amamentar. Para que isso seja possível, esse apoio perpassa pelas legislações e políticas de proteção, promoção e apoio à trabalhadora que amamenta $\mathrm{e}$ pelo conhecimento e cumprimento pelos gestores e colegas de trabalho. Além disso, a trabalhadora necessita de uma rede completa de apoio, dentro e fora do local de trabalho.

Reforça-se a necessidade de melhorias nas legislações e mobilização por parte de toda sociedade e a realização de estudos com objetivo de aperfeiçoar o apoio à amamentação nos ambientes de trabalho. 


\section{REFERÊNCIAS}

1. Palmer G. The politics of breastfeeding. London: Pandora Press, 1988.

2. Silva EA, Almeida SSC. Woman and work in the contemporary world: space conquest and implications for health-disease process. História e Cultura. 2017;6(3):306-24.

3. World Health Organization (WHO)/United Nations Children's Fund (UNICEF). Global Breastfeeding Scorecard: tracking progress for breastfeeding policies and programmes. 2017.

4. Rollins NC, Bhandari N, Hajeebhoy N, Horton S, Lutter CK, Martines JC et al. Why invest, and what it will take to improve breastfeeding practices? Lancet. 2016, 387(10017): 491-504.

5. Silva MM, \& Monteiro JCS. Atuação dos profissionais de saúde na realização das ações pró-amamentação: revisão da literatura. Cogitare Enferm. 2014,19(1):153-8.

6. Mendes KDS, Silveira, RCDCP, Galvão CM. Revisão integrativa: método de pesquisa para a incorporação de evidências na saúde e na enfermagem. Texto \& contexto enferm. 2008,17(4):758-64.

7. Araújo VS, Medeiros APDS, Barros ADC, Braga LS, Trigueiro JVS, Dias MD. Early weaning: aspects of the experiences of informal workers. Referência. 2013,III Série(10):35-43.

8. Brasileiro AA, Ambrosano GMB, Marba STM, Possobon RDF. A amamentação entre filhos de mulheres trabalhadoras. Rev. Saúde Públ. 2012,46(4):642-8.

9. Senarath U, Dibley MJ, Agho KE. Factors associated with nonexclusive breastfeeding in 5 east and southeast Asian countries: a multilevel analysis. J Hum Lact; 2010,26(3):248-57.

10. Chang SM, Rowe J, Goopy S. Non family support for breastfeeding maintenance among career women in Taiwan: A qualitative study. Int J Nurs Pract. 2014,20(3):293-301.

11. Tarrant M, Fong DY, Wu KM, Lee IL, Wong EM, Sham A, et al. Breastfeeding and weaning practices among Hong Kong mothers: a prospective study. BMC Pregnancy Childbirth. 2010,10(1):1-12.

12. Skafida V. Juggling work and motherhood: the impact of employment and maternity leave on breastfeeding duration: a survival analysis on Growing Up in Scotland data. Matern Child Health J. 2012,16(2):519-27.

13. Tsai SY. Impact of a breastfeeding-friendly workplace on an employed mother's intention to continue breastfeeding after returning to work. Breastfeed Med. 2013,8(2):210-6.

14. Bai YK, Wunderlich SM, Weinstock M. Employers' readiness for the mother friendly workplace: an elicitation study. Matern Child Nutr. 2012,8(4):483-91.

15. Chow T, Smithey Fulmer I, Olson BH. Perspectives of managers toward workplace breastfeeding support in the state of Michigan. J Hum Lact. 2011,27(2), 138-146.

16. Mandal B, Roe BE, Fein SB. The differential effects of fulltime and part-time work status on breastfeeding. Health Policy. 2010,97(1):79-86.

17. Ogbuanu C, Glover S, Probst J, Liu J, Hussey J. The effect of maternity leave length and time of return to work on breastfeeding. Pediatrics. 2011,127(6):1414-27.

18. Hojnacki SE, Bolton T, Fulmer IS, Olson BH. Development and piloting of an instrument that measures company support for breastfeeding. J Hum Lact. 2012,28(1):20-7.

19. Cooklin AR, Rowe HJ, Fisher JR. Paid parental leave supports breastfeeding and mother infant relationship: a prospective investigation of maternal postpartum employment. Aust N Z J Public Health. 2012,36(3):249-56.

20. Heymann J, Raub A, Earle A. Breastfeeding policy: a globally comparative analysis. Bull World Health Org Suppl. 2013,91(6):398-406.

21. Abdulloeva S, Eyler AA. Policies on worksite lactation support within states and organizations. J Womens Health. 2013,22(9):769-74.

22. Vitor RS, Vitor MDCS, de Oliveira TM, Corrêa CA, Menezes HS. Aleitamento materno exclusivo: análise desta prática na região Sul do Brasil. Rev. AMRIGS. 2010,54(1):44-8.

23. Brecailo MK, Corso ACT. Fatores associados ao aleitamento materno exclusivo em Guarapuava, Paraná. Rev. Nutr. 2010, 23(4):553-63.

24. Nguyen TT, Hawkins SS. Current state of US breastfeeding laws. Matern Child Nutr. 2013,9(3):350-8.

25. Rodrigues NA, Gomes ACG. Aleitamento materno: fatores determinantes do desmame precoce. Enferm. Rev. 2014,17(1):30-48.

26. Batista MR, Veleda AA, Coelho DF, Cordova FP. Orientações de profissionais da saúde sobre aleitamento materno: o olhar das puérperas. J Nurs Health. 2017,07(1):25-37.

27. Ribeiro KV, Florentino CLV, Mariano DCA, Peres PLP, Rodrigues BMRD. A amamentação e o trabalho informal: a vivência de mães trabalhadoras. Rev. Pró-UniverSUS. 2017,08(2):03-09.

28. Fernandes VMB, Santos EKA, Zampieri MFM, Gregório VRP, Hernandes MJ, Ribeiro LC. Condutas de gestores relacionadas ao apoio ao aleitamento materno nos locais de trabalho. Texto \& contexto enferm. 2018,27(3):1-12.

29. Ministério da Saúde. Agência Nacional de Vigilância Sanitária. Guia para implantação de salas de apoio à amamentação para a mulher trabalhadora. Brasília. 2015.

30. Brasil. Constituição da República Federativa do Brasil. Diário Oficial da União, 1988.

31. Brasil. Lei $n^{\circ} 11.770$, de 9 de setembro de 2008. Cria o Programa Empresa Cidadã, destinado à prorrogação da licença-maternidade mediante concessão de incentivo fiscal. Diário Oficial da União, 2008.

32. Brasil. Decreto $n^{\circ} 6.690$, de 11 de dezembro de 2008. Institui o Programa de Prorrogação da Licença à Gestante e à Adotante, estabelece os critérios de adesão ao Programa e dá outras providências Diário Oficial da União, 2008.

33. Brasil. Decreto-lei n 5.452, de 01 de maio de 1943. Consolidação das Leis do Trabalho (CLT). Diário Oficial da União, 1943. 